Published online 2017 April 13.

Abstract

\title{
Future Outcome of Sonography BI-RADS Category 3 Lesions
}

\author{
Afsaneh Alikhassi ${ }^{1, *}$ \\ ${ }^{1}$ Tehran University of Medical Sciences, Tehran, Iran \\ "Corresponding author: Afsaneh Alikhassi, Tehran University of Medical Sciences, Tehran, Iran. E-mail: afsanehalikhassi@yahoo.co.uk
}

Received 2016 December 21; Accepted 2017 February 08.

\section{Abstract}

Objectives: This study aims to find the frequency and the malignancy rate of BI-RADS category 3 lesions detected on screening breast ultrasound and to evaluate whether they fullfilled the requirements of the latest American college of radiology imaging and reporting system.

Methods: Of 500 asymptomatic women who came to our center for screening sonography themselves or referred by their clinician, patients for whom BI-RADS category 3 lesions were seen on the ultrasound were selected. Clinical outcome of these lesions using one year follow up, biopsy or a combination of both were evaluated.

Results: The frequency of BI-RADS category 3 lesions detected on screening ultrasound was $18 \%$ (90/500). Of these patients, one was eventually proven to have malignancy with malignancy rate of $1.1 \%$. This detected malignancy was early breast cancer with no lymph node metastasis.

Conclusions: Although the frequency of ultrasound BI-RADS category 3 lesions is considerably high (18 \%), the malignancy rate is low (1.1\%). Therefore careful assessment and follow up of BI-RADS category 3 can avoid unnecessary biopsy or surgery.

This is an abstract presented in the 33rd Iranian congress of radiology (ICR) and the 15th congress of Iranian radiographic science association (IRSA) 